\title{
Recent Regulatory Amendment in Schedule Y: Impact on Bioequivalence Studies Conducted In India
}

\author{
Manoj Karwa ${ }^{1 *}$, Saurabh Arora $^{2}$ and Shilpa Garg Agrawal ${ }^{3}$ \\ ${ }^{1}$ Head-PPM, Auriga Research Ltd., New Delhi, India \\ ${ }^{2}$ Managing Director, Auriga Research Ltd., New Delhi, India \\ ${ }^{3}$ Founder \& CEO, SenseCR, Mumbai, India
}

\begin{abstract}
Bioequivalence studies are conducted for comparison of two medicinal products containing the same active ingredients, and these studies are mostly conducted in healthy subjects. The regulatory guidelines in terms of serious adverse events (SAEs) reporting, informed consent, compensation in case of injury or death for bioequivalence studies are similar as in clinical trials. Recently there have been three amendments in Schedule $Y$ of the Drugs and Cosmetics Act. The first gazette notification is G.S.R. 53 (E) dated January 30, 2013, with insertion of Rule $122 \mathrm{DAB}$ which specifies procedures to analyze the reports of SAEs occurring during clinical trials and payment of compensation in case of trial related injury or death as per defined timelines. The detailed process is described by addition of Appendix XII in Schedule Y. The second gazette notification is G.S.R. 63(E) dated February 01, 2013 with insertion of Rule 122 DAC which specifies conditions under which application for conduct of clinical trials shall be approved by licensing authority, which includes a very important point that the sponsors, their subsidiaries, agents, sub-contractors, and clinical trial sites shall allow inspectors authorized by CDSCO to inspect their premises. The third amendment is related to mandatory registration of the Ethics Committees (EC) in the Drug and Cosmetic act vide G.S.R. 72(E) dated February 08, 2013 with addition of Rule 122 DD.
\end{abstract}

Keywords: Bioequivalence; Schedule Y; Regulatory; SAE; Ethics committees; Informed consent; Compensation; India

\section{Serious Adverse Event Reporting and Compensation: Rule 122 DAB of Schedule Y}

As defined in schedule Y, SAE is an untoward medical occurrence during clinical trial that is associated with death, in patient hospitalization (in case the study was being conducted on out-patient), prolongation of hospitalization (in case the study was being conducted on in-patient), persistent or significant disability or incapacity, a congenital anomaly or birth defect or is otherwise life threatening [1].

The reporting timeline for all serious and unexpected adverse events by principal investigator is now within 24 hours of occurrence of event to the Licensing Authority, sponsor and the EC; whereas, prior to this amendment, the 24 hours reporting was limited to sponsor by the investigator. The detailed report of SAEs, after due analysis, should be forwarded by the investigator and sponsor to Chairman of the EC, Licensing Authority and the Head of the Institution within ten calendar days of occurrence of the SAEs. The report of SAEs of death, in addition, also needs to be forwarded to the Chairman of expert committee appointed by Licensing Authority [1].

Prior to the amendment, the SAEs management and reporting was done by Bioequivalence centers, but now sponsor also need to send a separate report after due analysis by their medical affairs team in cases of SAEs.

Serious adverse event reports submitted to Licensing Authority should be in color coded binding, where the reports of SAEs of deaths are submitted in red cover, the reports of SAEs of injury other than deaths in blue cover and the remaining cases of SAE reports in white cover [2]. We have observed that most of the SAEs submitted by bioavailability and bioequivalence (BA/BE) centers are in white cover, as adverse events are already documented for generic products and can be medically managed.
In case of any $\mathrm{AE}$ (adverse events) during the $\mathrm{BA} / \mathrm{BE}$ study, subjects need to be provided free medical management as long as required and the expenses have to be borne by the sponsor of the study. In addition, sponsor or his representative needs to compensate subjects if the injury is clinical trial related, due to adverse effect of investigational product, violation of the approved protocol, scientific misconduct or negligence by the sponsor or his representative or by investigator, failure of investigational product to provide intended therapeutic effect, use of placebo in a placebo controlled trial, adverse effects due to concomitant medications, injury to a child in-utero due to participation of parents, or due to any clinical trial procedures [1]. Some of the above cases are not applicable to BA/BE studies like use of placebo and injury to child in-utero because these studies are conducted with marketed comparator and in healthy subjects only. Further BA/BE study centers already follow the practice of providing medical management of all AEs and SAEs whether related or not related. However, clarity on compensation amount in case of death or injury for healthy volunteer is required from the licensing authority.

The final authority for determination of the cause of death or injury and also the quantum of compensation is now the Licensing Authority, who will communicate the compensation amount to sponsor within three months of receiving the report of SAE of death or injury. In case of SAEs of death, the expert committee shall provide

*Corresponding author: Manoj Karwa, Clinical Research Department, Auriga Research Ltd., 3/15 Kirti Nagar Industrial Area, New Delhi - 110015, India, Tel: +911145754546-47; E-mail: manojkarwa@aurigaresearch.com

Received May 26 2013; Accepted June 25, 2013; Published July 01, 2013

Citation: Karwa M, Arora S, Agrawal SG (2013) Recent Regulatory Amendment in Schedule Y: Impact on Bioequivalence Studies Conducted In India. J Bioequiv Availab 5: 174-176. doi:10.4172/jbb.1000154

Copyright: (C) 2013 Karwa M, et al. This is an open-access article distributed under the terms of the Creative Commons Attribution License, which permits unrestricted use, distribution, and reproduction in any medium, provided the original author and source are credited. 
its recommendation about causality and quantum of compensation to the licensing authority, and then the licensing authority shall pass the final order [1].

The sponsor has to pay the compensation in case of clinical trial related injury or death within thirty days of the receipt of order from Licensing Authority. In case the sponsor fails to provide medical management and / or financial compensation, the Licensing Authority may take necessary action as per rule, including suspension or cancellation of the clinical trial and/or restrict sponsor including his representative(s) to conduct any further clinical trials in India [1].

These changes have also led to requirement of changes in the Informed Consent Documents (ICD) where additional information should be incorporated mentioned in amendments. As per the new requirements in Appendix V of Schedule Y, now, the ICD should clearly state that the subject is entitled to free medical management as long as required in case of injury, and financial compensation in case of clinical trial related injury or death. The investigator will have to clearly inform the subject about his right to claim compensation in case of trial related injury or death, and to contact the sponsor / representative directly for any claim related queries. The contact details of sponsor representative should be provided in the ICD. In order to aid the calculation of compensation amount, the ICD now should have further details about the subject like qualification, occupation, annual income, address, and contact details of the nominee and his/her relation with the subject. A copy of ICD should be provided to subject and same should be mentioned in the ICD document [1].

\section{Permission to Conduct Clinical Trials: Rule 122 DAC of Schedule Y}

Rule 122 DAC basically discusses about the compliance to regulatory and ethical guidelines for data submitted for clinical trials, and actions taken in case of noncompliance. Licensing Authority may inspect sponsor including their employees, subsidiaries, and branches, their agents, contractors, subcontractors, clinical trial sites, $\mathrm{BA} / \mathrm{BE}$ centers and investigators to verify the compliance and they should provide adequate replies to the inspectors in relation to conduct of clinical studies. Licensing Authority may reject or cancel studies and debar investigators, sponsors including their employees, subsidiaries and branches, their agents, contractors and subcontractors for conduct of any clinical trials in future [3]. It is very important that each stakeholder understands his/ her responsibility and complies strictly with the regulatory requirements where need of understanding of good clinical practice (GCP) and training arises for all stakeholders.

\section{Ethics Committee Registration and Functioning: Rule 122 DD of Schedule Y}

With introduction of Rule 122 DD in Schedule Y, prior registration of ECs with DCGI is mandatory to review and accord its approval to a clinical trial protocol. The registration is given for a period of three years to the committees from the date of issue, unless suspended or cancelled [4]. Now all BA/BE study protocols need to be reviewed and approved by a registered Independent EC from same location as that of the $\mathrm{BA} / \mathrm{BE}$ center.

Of the additional responsibilities given to the Ethics Committees, one of the major responsibility is forwarding it's report on the serious adverse event of injury, after due analysis with causality assessment and opinion on financial compensation to the licensing authority within twenty one calendar days of the occurrence of the event, and in cases of SAE of death report should also be sent to Chairman of the expert committee [5]. The expert committee and licensing authority are mainly considering the EC's opinion to decide on the relatedness and quantum of compensation. Currently there is lack of clarity from CDSCO on guidelines for calculation of compensation amount; the ECs are thus having a tough time giving their opinion on compensation amount. ECs are now required to maintain all clinical trial documents reviewed by them for at least five years and be open for inspections by licencing authority at the EC office.

So far, about 500 ECs have been registered with CDSCO and about 60 of them are independent ECs [6]. The registration of independent ECs has been initiated after a long waiting period and many of them got query letter due to stringent scrutiny of their applications. But the independent ECs can review only the study protocols and related documents of BA/BE studies of approved products. So BA/BE centers now need to approach institutional ECs for approval of BE study protocols of drug products not yet approved in India. Also, many BA/ $\mathrm{BE}$ centers also conduct phase I pharmacokinetic studies along with BA/BE studies. Even for such phase I studies, the BA/BE centers will have to approach Institutional ECs, since Independent ECs will not be able to take up review of such studies. The biggest challenge will arise when the institutional ECs also reject such proposals which are not conducted in their institute / site.

\section{Impact of Current Regulatory Changes on BA/BE Industry of India}

The requirements for $\mathrm{BA} / \mathrm{BE}$ studies are becoming similar as for clinical trials and pose challenges in front of $\mathrm{BA} / \mathrm{BE}$ centers. The regulatory authority may start asking statistical justification for number of subjects in BE study. Many processes, which earlier only required notification now need approval, for e.g., all protocol amendments, change of $\mathrm{BA} / \mathrm{BE}$ study center. Earlier, $\mathrm{BE}$ for export studies were approved for the products which are even not approved and marketed in India but it may not be the case now. There is need to revise the existing SOPs and other study BA/BE documents, as per the new amendments for SAEs' reporting timelines and compensation. This may lead to some delays in the completion of the existing studies.

However, considering the slowdown in the global clinical trials, the Indian pharma industry will again focus on generic business and number of BA/BE studies may increase both for registration in India and also for export.

\section{Conclusion}

With the amendments to Schedule Y, currently, the entire clinical research industry, including the $\mathrm{BA} / \mathrm{BE}$ centers are experiencing a tough time due to lack of clarity on many aspects of these amendments.

There is more clarity expected from the DCGI on the definition of injury, reasons which are included in clinical trial related injury, calculation of compensation amount and compensation in cases where the injury or death is not related to clinical trial. Once this clarity is received from DCGI, hopefully the current turbulence in the Indian clinical research industry will settle down and the conduct of BA/ $\mathrm{BE}$ studies would be more streamlined, with stricter vigilance and compliance.

\section{References}

1. Compensation to be provided during clinical trial. Central Drugs Standard Control Organization website. New Delhi, India: Directorate General of Health Services, Ministry of Health and Family Welfare, Government of India. 
Citation: Karwa M, Arora S, Agrawal SG (2013) Recent Regulatory Amendment in Schedule Y: Impact on Bioequivalence Studies Conducted In India. J Bioequiv Availab 5: 174-176. doi:10.4172/jbb.1000154

2. System of pre-screening for submission of reports of SAEs to Central Drugs Standard Control Organization website. New Delhi, India: Directorate General of Health Services, Ministry of Health and Family Welfare, Government of india. Accessed on June 16, 2013.

3. Permission to conduct clinical trials. Central Drugs Standard Control Organization website. New Delhi, India: Directorate General of Health Services, Ministry of Health and Family Welfare, Government of India.

4. System for the pre-screening of the applications for registration of Ethics committee. Central Drugs Standard Control Organization website. New Delhi,
India: Directorate General of Health Services, Ministry of Health and Family Welfare, Government of India.. Accessed on June 16, 2013.

5. Registration requirement for Ethics Committees. Central Drugs Standard Control Organization website. New Delhi, India: Directorate General of Health Services, Ministry of Health and Family Welfare, Government of India. Accessed on June 16, 2013.

6. List of registered Ethics Committees. Central Drugs Standard Contro Organization website. New Delhi, India: Directorate General of Health Services, Ministry of Health and Family Welfare, Government of India. Accessed on June 16, 2013. 